\title{
The nightside magnetic field line open-closed boundary and polar rain electron energy-latitude dispersion
}

\author{
S. Wing and Y. L. Zhang \\ The Johns Hopkins University Applied Physics Laboratory, Laurel, Maryland, USA \\ Correspondence to: S. Wing (simon.wing@jhuapl.edu)
}

Received: 6 October 2014 - Revised: 24 November 2014 - Accepted: 24 November 2014 - Published: 12 January 2015

\begin{abstract}
The polar rain electrons near the open-closed field line boundary on the nightside often exhibit energy-latitude dispersion, in which the energy decreases with decreasing latitude. The solar wind electrons from the last open-field line would $\boldsymbol{E} \times \boldsymbol{B}$ drift equatorward as they move toward the ionosphere, resulting in the observed dispersion. This process is modeled successfully by an open-field line particle precipitation model. The existing method for determining the magnetotail $X$ line distance from the electron dispersion underestimates the electron path length from the $X$ line to the ionosphere by at least $33 \%$. The best estimate of the path length comes from using the two highest energy electrons in the dispersion region. The magnetic field line open-closed boundary is located poleward of the highest energy electrons in the dispersion region, which in turn is located poleward of Defense Meteorological Satellite Program (DMSP) b6, b5e, and b5i boundaries. In the four events examined, b6 is located at least $0.7-1.5^{\circ}$ equatorward of the magnetic field line open-closed boundary. The energy-latitude dispersion seen in the electron overhang may result from the plasma sheet electron curvature and gradient drifts into the newly closed field line.
\end{abstract}

Keywords. Magnetospheric physics (polar cap phenomena)

\section{Introduction}

Magnetic reconnection is an important process in space and plasma physics. On the dayside, magnetic reconnection between the interplanetary magnetic field (IMF) and the magnetospheric magnetic field lines causes the closed magnetospheric field lines to become open. These open field lines at both the Northern Hemisphere and Southern Hemisphere are carried by the solar wind to the nightside, where they recon- nect to form a closed field line in the magnetotail (Dungey, 1961).

As a result of the magnetic reconnection on the dayside, the shocked solar wind can enter the magnetosphere and a fraction precipitates into the ionosphere. The solar wind entry points and the processes at the magnetopause lead to four types of particle precipitation regions: open-field lowlatitude boundary (LLBL), cusp, mantle, and polar rain (e.g., Newell et al., 1991; Wing et al., 1996, 2001). The polar rain occupies most of the polar cap and consists mainly of precipitating solar wind suprathermal electrons (Fairfield and Scudder, 1985; Wing et al., 1996).

The energy-latitude dispersion in the polar rain electron flux was sometimes observed near the nightside auroral oval by the Akebono and Defense Meteorological Satellite Program (DMSP) satellites (Shirai et al., 1997; Zhang et al., 2011). The red lines in Fig. 1 mark the poleward boundary of the energy-latitude dispersion in the DMSP observations. Shirai et al. (1997) attributed the energy-latitude dispersion to being a signature of the last injected solar wind suprathermal electrons before the open field-lines reconnect in the magnetotail and become closed. Because of the duskward crosstail electric field (e.g., Pedersen et al., 1985), these solar wind electrons from the last open-field line would undergo $\boldsymbol{E} \times \boldsymbol{B}$ equatorward convection as they move toward the ionosphere, leading to the observed energy-latitude dispersion. The polar rain electron energy dispersion has been used to estimate the electron path length from the $X$ line to the ionosphere and the $X$ line location in the magnetotail (Shirai et al., 1997; Alexeev et al., 2006; Zhang et al., 2011). This dispersion method seems reasonable in principle, but the validation of the method has been difficult because of the lack of coincident in situ reconnection measurements in the magnetotail. 


\section{DMSP observations of auroral oval and polar cap}
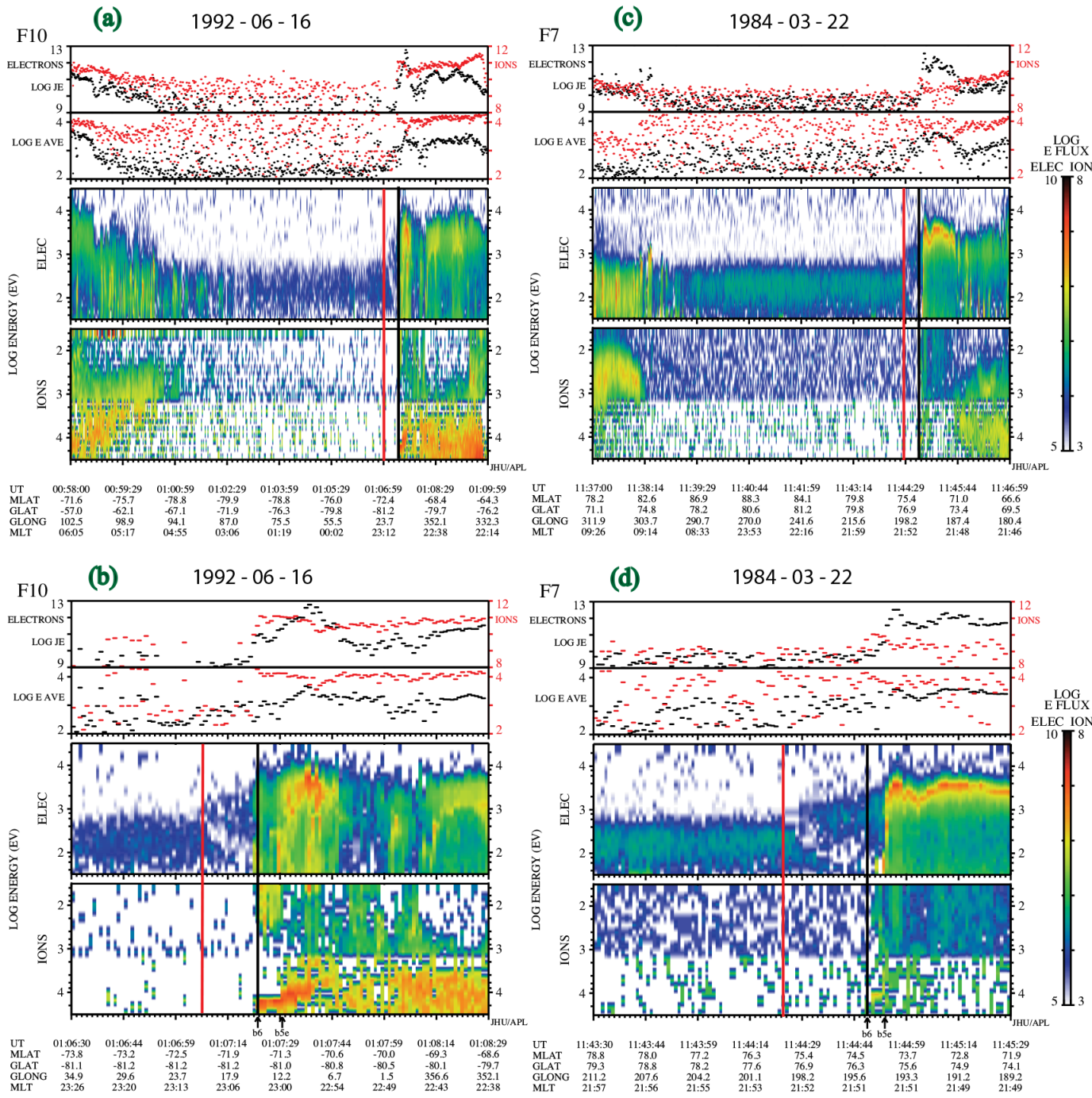

Figure 1. (a) and (c) DMSP observations of the auroral oval and polar cap. (b) and (d) zoom in on the nightside region where the polar rain meets the poleward edge of the auroral oval in (a) and (b), respectively. (b) and (d) show the polar rain energy-latitude dispersion, b6, and b5e. The vertical red lines indicate the location of the highest energy polar rain electrons in the dispersion region while the vertical black lines indicate $b 6$.

Newell et al. (1996) identified a few useful boundaries near the poleward edge of the nightside auroral oval, namely b5e, $\mathrm{b} 5 \mathrm{i}$, and b6. b5e and b5i indicate the region near the poleward boundary of the auroral oval where the precipitating electron and ion differential energy flux, respectively, drop off sharply, typically by an order of magnitude over a short distance $\left(<0.2^{\circ}\right)$. Usually, b5e and b5i are close to each other. b6 is defined as the poleward edge of the subvisual drizzle, which is the region of weak ion and electron precipitation typically poleward of both b5e and b5i. The locations of b6 and b5e in Fig. $1 \mathrm{~b}$ and $\mathrm{d}$ are indicated by the labels below the bottom panel. Studies have used b6 (e.g., Boakes et al., 2008; Longden et al., 2010) or a halfway point between b5 (either b5e or b5i) and b6 (Hubert et al., 2006) as the best available proxy for the magnetic field open-closed boundary. These studies also show that, in general, there are systematic discrepancies between the open-closed boundary obtained from DMSP and those obtained from optical images, but the exact causes of these discrepancies are not entirely clear.

At the Johns Hopkins University Applied Physics Laboratory (JHU/APL), Wing et al. (1996, 2001) developed an open-field line particle precipitation model (APL-OPM) to model precipitating ions and electrons in the open-field line LLBL, cusp, mantle, and polar rain. APL-OPM, which was developed for studying dayside particle precipitation, can be extended to model the nightside electron energy-latitude dis- 
persion. Because the nightside open-closed boundary (magnetotail $X$ line) is known and can even be arbitrarily set in APL-OPM, the model can be a useful tool to evaluate how well the polar rain dispersion can be used to estimate the $X$ line location.

In the present study, we model the polar rain electron energy dispersion using a modified APL-OPM (Wing et al., 1996, 2001). Based on this model, we examine the accuracy of the existing method for using the dispersion to estimate electron path length and $X$ line distance. Finally, we examine the issue of where the magnetic field line open-closed boundary is located based on our modeling and observational work.

\section{Modeling the polar rain energy-latitude dispersion}

\subsection{APL-OPM description}

APL-OPM has been fully described in Wing et al. (1996, 2001). Therefore, only a brief description is provided here. For a given IMF orientation, solar wind temperature, velocity, density, ionospheric convection speed, and dipole tilt angle, the model computes the phase space density of the precipitating ions and electrons in three steps. In the first step, the model traces the precipitating particles from the ionosphere back along the guiding centers to the magnetopause entry point using the T96 magnetic field model (Tsyganenko and Stern, 1996) and SuperDARN electric field (Ruohoniemi and Greenwald, 1996), assuming conservation of magnetic moment and negligible parallel electric field. In the second step, the model computes acceleration $(j \cdot \boldsymbol{E}>0)$ or deceleration $(j \cdot \boldsymbol{E}<0)$ imparted on the particles when they cross the magnetopause current layers from the magnetosheath to the magnetosphere (e.g., Cowley and Owen, 1989; Hill and Reiff, 1977). From this calculation, the model obtains the velocity that the particle originally has in the magnetosheath. Finally, it computes the phase space density of particles with that velocity using the gas-dynamics calculations of Spreiter and Stahara (1985) with the assumption that electrons have Maxwellian and ions have $\kappa$ distributions. In addition, APL-OPM imposes charge quasi-neutrality with a selfadjusting parallel electric field at the magnetopause and includes suprathermal electrons, which are crucial for polar rain modeling. Wing et al. $(1996,2001)$ showed that APLOPM can model not just the cusp but also open-field line LLBL, mantle, and polar rain particles.

\subsection{APL-OPM extension to the nightside}

In order to study the nightside polar rain near the openclosed boundary, we make the following modifications to APL-OPM. The magnetotail reconnection site (the last open-closed boundary) is arbitrarily set at $X=-50 R_{\mathrm{E}}$, which is the tail boundary of the model. All the field lines that cross the $Y-Z$ plane off the equatorial plane at $X=-50 R_{\mathrm{E}}$ are considered open and filled with solar wind suprathermal electrons, which are assumed to enter the magnetosphere at $X<-50 R_{\mathrm{E}}$ and travel to $X=-50 R_{\mathrm{E}}$ unmodified. These assumptions are supported by observations. For example, Fig. 1 shows that the nominal polar rain electrons on the nightside have fairly uniform energy fluxes (Newell et al., 1996), suggesting that the magnetosheath and magnetopause properties as well as the processes at the entry point are fairly uniform at the entry points at these locations in the deep tail. Note that this choice of the $X$ line might not and most likely would not be consistent with the SuperDARN electric field, but it is not the goal of the present study to model a specific observation. We only require that the electric field has a significant duskward component, which it typically does (e.g., Pedersen et al., 1985). In order to accentuate the polar rain energy-latitude dispersion, the SuperDARN nightside potential is multiplied by an arbitrary factor of 10 (any reasonably large duskward electric field would work for the purpose of the present study). In mapping the electric field from the ionosphere to the magnetosphere, we assume that the parallel electric field is negligible.

As in our previous APL-OPM runs, we set the altitude of "detected" particle at $1.13 R_{\mathrm{E}}$, which corresponds to the typical DMSP spacecraft altitude. The model DMSP trajectory is set to nearly noon-meridian orbit. We trace 19 electrons and ions with energies from $32 \mathrm{eV}$ to $30 \mathrm{keV}$ in 19 logarithmically equally spaced steps, the same energies as the 19 electron and ion channels in the DMSP SSJ4 instrument (Hardy et al., 1986). The magnetic coordinates used for displaying the DMSP and model calculations are the altitude-adjusted corrected geomagnetic (AACGM) coordinates (Baker and Wing, 1989).

The present study investigates only the nightside polar rain electrons, which mainly consist of solar wind suprathermal electrons. We ran the model with the following input parameters: $\operatorname{IMF}\left(B_{x}, B_{y}, B_{z}\right)=(-3.4,-0.5,-12.3) \mathrm{nT}$, solar wind thermal $n=11 \mathrm{~cm}^{-3}, T_{\mathrm{i}}=1 \times 10^{5} \mathrm{~K}(8.6 \mathrm{eV}), T_{\mathrm{e}}=3 \times 10^{4} \mathrm{~K}$ (2.6 eV), $V=400 \mathrm{~km} \mathrm{~s}^{-1}$, suprathermal (halo) electron $n_{\mathrm{s}}=$ $0.2 \mathrm{~cm}^{-3}, T_{\mathrm{s}}=1 \times 10^{6} \mathrm{~K}(86 \mathrm{eV}), \kappa=7$. The electric field is obtained from SuperDARN convection pattern for strongly southward IMF (Ruohoniemi and Greenwald, 1996). This is essentially the same run for the strongly southward IMF case presented in Wing et al. (2001), except that here we extend the run to the nightside, whereas Wing et al. (2001) only presents the model calculation for the dayside. Figure 2 shows the model calculation of the polar rain electron spectra along the model DMSP path. The model calculation for the dayside showing particle precipitation in the open-field line LLBL, cusp, and mantle is shown in Fig. 2 in Wing et al. (2001) and is not shown here. In Fig. 2, the spectra between 12:11:40 and 12:11:47 universal time (UT) represent typical spatially uniform polar rain electrons. The small fluxes seen at 12:11:55 UT are just numerical artifacts. The energy-latitude dispersion can be seen between 


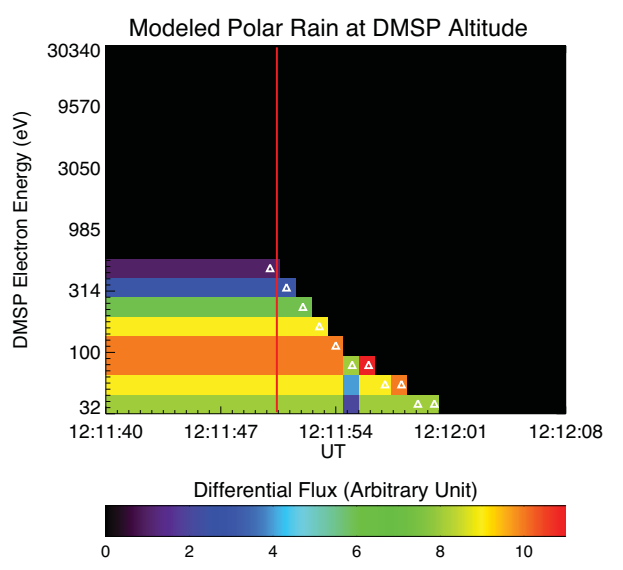

Figure 2. Polar rain electron dispersion from the APL-OPM model calculation. The electron spectra between 12:11:40 and 12:11:49 UT represent the typical polar rain. There is a clear electron energy-latitude dispersion after 12:11:49 UT. The white triangles, which indicate the highest energy detected at a given latitude, give a measure of the energy-latitude dispersion. They are used to determine the electron path lengths between DMSP altitude and magnetotail reconnection site. The vertical red line marks the location of the highest energy polar rain electrons in the dispersion region.

12:11:50 and 12:12:01 UT, where the highest electron energy for each spectrum or latitude, which is marked by the white triangle, decreases with decreasing latitude. The last openfield line is encountered slightly before 12:11:50 UT, which marks the time when the highest energy electron arrives in the ionosphere. After entering the magnetosphere, because of the dominant duskward electric field, the electrons undergo $\boldsymbol{E} \times \boldsymbol{B}$ equatorward convection as they move to the ionosphere. Hence, lower-energy electrons arrive at successively lower latitudes in the ionosphere. This energy-latitude dispersion is analogous to the dayside cusp ion dispersion resulting from the dayside reconnection, except that because of the $\boldsymbol{E} \times \boldsymbol{B}$ poleward convection in the cusp, the energy decreases with increasing latitude (e.g., Wing et al., 1996, 2001). This polar rain electron energy dispersion has been previously observed (Shirai et al., 1997; Zhang et al., 2011).

This simple model calculation successfully demonstrates quantitatively that the magnetotail reconnection and plasma $\boldsymbol{E} \times \boldsymbol{B}$ equatorward convection can lead to the polar rain energy-latitude dispersion near the open-closed boundary.

\section{Estimation of electron path length}

\subsection{Estimation of electron path length in the model}

In the model, the electron path length from the reconnection site to the polar ionosphere can be calculated. In general, the path length has a dependence on the energy. The lower-energy electrons would have more time to $\boldsymbol{E} \times \boldsymbol{B}$ drift to lower latitudes that have shorter magnetic field lines, resulting in shorter path lengths. For example, the $462 \mathrm{eV}$ electrons (marked by the top or first white triangle in Fig. 2) have a path length of $64 R_{\mathrm{E}}$, whereas the $32 \mathrm{eV}$ electrons (the bottom triangles in Fig. 2) have a path length of $56 R_{\mathrm{E}}$. The direct straight line distance $\left(50 R_{\mathrm{E}}\right)$ between the center of the Earth and the $X$ line is about $78 \%$ of the path length $S\left(64 R_{\mathrm{E}}\right)$ of the highest energy electron. This difference can be attributed to the curvature of the magnetic field lines and cross-field drift of the electrons.

A method for estimating the electron path length based on the polar rain electron energy dispersion has been developed (Shirai et al., 1997; Alexeev et al., 2006). The method is briefly summarized here. An electron with a constant velocity $V_{1}$ would take time $\left(t_{1}-t_{0}\right)$ to travel a distance $S_{1}$ (see Eq. 1), where $t_{0}$ is the time of the reconnection (the last solar wind electron injection), $t_{1}$ is the time for the electron to be observed in the ionosphere (e.g., by DMSP satellite), and $S_{1}$ is electron path length from $X$ line to the ionosphere. Equation (2) applies to an electron with a different velocity $\left(V_{2}\right)$. $S_{1}$ is not usually the same as $S_{2}$.

$t_{1}-t_{0}=\frac{S_{1}}{V_{1}}$
$t_{2}-t_{0}=\frac{S_{2}}{V_{2}}$

While $t_{1}, t_{2}, V_{1}$, and $V_{2}$ can be measured by low-altitude satellites, $t_{0}, S_{1}$, and $S_{2}$ are unknown. Equations (1) and (2) are not sufficient to solve the three unknown parameters. Hence, an assumption is needed. If we assume that $S_{1} \approx S_{2}=S$ and plasma is frozen in, we obtain Eq. (3),

$\Delta t=t_{1}-t_{2}=S\left(\frac{1}{V_{1}}-\frac{1}{V_{2}}\right)$,

where $\Delta t=$ the difference of the arrival time between the two electrons.

Because of nonzero $\boldsymbol{E} \times \boldsymbol{B}$ drift, within $\Delta t$ the electrons also move a distance $L$ in the direction of $\boldsymbol{E} \times \boldsymbol{B}$ drift at the ionosphere altitude. So, $\Delta t=L / V_{\mathrm{p}}$, where $V_{\mathrm{p}}$ is the $\boldsymbol{E} \times \boldsymbol{B}$ drift speed. However, electron observations are usually made from satellites moving at speed $V_{\mathrm{s}}$. Hence, $\Delta t$ in the satellite reference frame $\left(\Delta t^{*}\right)$ can be expressed as $\Delta t^{*}=L / V_{\mathrm{s}}$, assuming that the $X$ line location and reconnection rate are steady or quasi-steady for at least several seconds. Therefore, $\Delta t=\left(V_{\mathrm{s}} / V_{\mathrm{p}}\right) \Delta t^{*}$ for the case where $V_{\mathrm{s}}$ is parallel to $V_{\mathrm{p}}$. If $V_{\mathrm{s}}$ and $V_{\mathrm{p}}$ are not parallel, then a component of $V_{\mathrm{p}}$ that is parallel to $V_{\mathrm{S}}$ would be used instead. By replacing $\Delta t$ in Eq. (3), we obtain the expression for the electron path length $(S)$ :

$S=\frac{\left(\frac{V_{\mathrm{s}} \Delta t^{*}}{V_{\mathrm{p}}}\right)}{\left(\frac{1}{V_{1}}-\frac{1}{V_{2}}\right)}$.

This equation is similar to the one used by Alexeev et al. (2006). In Eq. (4), the error in $V_{\mathrm{p}}$ is a major source of the 
Table 1. Dispersion time, energy, and electron path lengths estimated by Eq. (4) in the model run shown in Fig. 2 (see text). The fifth row lists the electron path lengths calculated from the given electron pairs.

\begin{tabular}{lcccccc}
\hline Position & First & Second & Third & Fourth & Fifth & Sixth \\
\hline Time (UT) & $12: 11: 50$ & $12: 11: 51$ & $12: 11: 52$ & $12: 11: 53$ & $12: 11: 54$ & $12: 11: 55$ \\
Time relative to first electron (s) & 0 & 1 & 2 & 3 & 4 & 5 \\
Energy (eV) & 462 & 314 & 215 & 147 & 100 & 69 \\
Path length $\left(R_{\mathrm{E}}\right)$ & & 43 & 39 & 36 & 32 & 29 \\
Electron pair & & (first, second) & (first, third) & (first, fourth) & (first, fifth) & (first, sixth) \\
\hline
\end{tabular}

uncertainty in $S$ because $V_{\mathrm{s}}, \Delta t, V_{1}$, and $V_{2}$ can usually be accurately determined. Equations (3) and (4) are derived with the assumption $S_{1}=S_{2}=S$, which also introduces some inaccuracies.

The model is run with $V_{\mathrm{s}}=7.8 \mathrm{~km} \mathrm{~s}^{-1}$, which is the DMSP satellite speed, and $V_{\mathrm{p}}=\boldsymbol{E} \times \boldsymbol{B}$ drift $=1.7 \mathrm{~km} \mathrm{~s}^{-1}$, where $\boldsymbol{E}$ is the same as the model $\boldsymbol{E}$ described in Sect. 2.2 and $\boldsymbol{B}$ is obtained from the International Geomagnetic Reference Field (IGRF) at the DMSP satellite location. Using Eq. (4), we calculate $S$ for five pairs of selected triangles in Fig. 2: (first, second), (first, third), (first, fourth), (first, fifth), and (first, sixth). Table 1 shows the time (UT) of these selected points, their energies, and the resulting electron path lengths $(S)$. There is a trade-off in errors of using a pair of electrons that are close vs. far apart in energy. Using two electrons having a large energy difference, e.g., the pair (first, sixth) in Table 1, would reduce the error in $L$ and $\Delta t^{*}$ but would decrease the validity of the assumption of $S_{1}=S_{2}=S$. The latter generally turns out to be a more dominant source of error. Table 1 shows that $S$ ranges from 43 to $29 R_{\mathrm{E}}$ and averages to $36 R_{\mathrm{E}}$, which are much shorter than the actual path lengths as determined from electron tracing in the model (64-56 with an average of $60 R_{\mathrm{E}}$ ). Hence, the calculation here shows that the method can underestimate the actual electron path length on average by about $40 \%$. The best result comes from using the highest two energies, pair (first, second), which results in $S=43 R_{\mathrm{E}}$ and which underestimates the actual electron path length $\left(64 R_{\mathrm{E}}\right)$ by $33 \%$.

The underestimation error would decrease with increasing electron energy and vice versa. As discussed in the beginning of this section, $S$ decreases when lower energy electrons are used. Hence, using a pair of lower energy electrons even if they are from adjacent energy channels (minimizing $\Delta S=$ $\left|S_{1}-S_{2}\right|$ ) could lead to a larger error than using pair (first, second). For example, $S$ for the pairs (second, third), (third, fourth), and (fourth, fifth) are 36, 30, and $24 R_{\mathrm{E}}$, respectively. For the same reason, we have not used the last five triangles in Fig. 2, which would result in smaller electron path lengths and larger error than pair (first, second).

\subsection{A couple examples from DMSP observations}

To illustrate, we apply the above method to two real DMSP dispersion events: one for weakly northward and one for strongly southward IMF. We choose these two events because (1) we would like to show that even with unusually large polar rain electron energies, up to a few $\mathrm{keV}$, the energy-latitude dispersion can still be observed and (2) higher energy would give more accurate estimates of the path length $S$ and $X$ line distance. Figure 3a shows the polar rain dispersion event on 17 April 2002. The associated $\operatorname{IMF}$ is $\operatorname{GSM}(X, Y, Z)=(-6,13,1) \mathrm{nT}$, indicating a weakly northward IMF $B_{z}$ and a dominant IMF $B_{y}$. As discussed in Sect. 3.1, the method would give the most accurate estimate of $S$ by using the two highest energy electrons (the top two triangles), which correspond to (19:48:23, 19:48:24) UT and electron energies $(3040,1393)$ respectively. Using Eq. (4), $V_{\mathrm{s}}=7.8 \mathrm{~km} \mathrm{~s}^{-1}$ (DMSP satellite speed), and ionospheric plasma drift speed along DMSP path $V_{\mathrm{p}}=1.73 \mathrm{~km} \mathrm{~s}^{-1}(\mathrm{Su}-$ perDARN convection map (Ruohoniemi and Greenwald, 1996) and DMSP SSIES observations), we obtain path length $S=49 R_{\mathrm{E}}$. However, if the method underestimates $S$ by $33 \%$ as obtained by the APL-OPM run in Sect. 3.1, the actual $S$ is probably closer to $73 R_{\mathrm{E}}$. Assuming the same ratio ( $\sim 78 \%$ ) between the reconnection $X$ line location $\left(50 R_{\mathrm{E}}\right)$ and the electron path length $\left(64 R_{\mathrm{E}}\right)$ obtained in the APLOPM model run in Sect. 3.1, the $X$ line location is estimated at $X=78 \% \times\left(-73 R_{\mathrm{E}}\right)=-57 R_{\mathrm{E}}$.

Figure $3 \mathrm{~b}$ shows another example of polar rain electron dispersion on 2 August 2002. The two highest energy electrons (the top two triangles) correspond to (01:25:14, $01: 25: 15)$ and electron energies $(1393,640)$ respectively. The IMF condition for this event is $\operatorname{GSM}(X, Y, Z)=(1.5,-10$, $-11) \mathrm{nT}$, indicating a strongly southward IMF. Using the method, we obtained electron path length $S=74 R_{\mathrm{E}}$, but the actual $S$ may be closer to $111 R_{\mathrm{E}}$, assuming the same $33 \%$ underestimation error obtained in Sect. 3.1. The $X$ line location is estimated at $X=78 \% \times\left(-111 R_{\mathrm{E}}\right)=-87 R_{\mathrm{E}}$.

\section{Where is the nightside magnetic field line open-closed boundary?}

Many studies used the poleward edge of the subvisual drizzle (b6) or poleward edge of the auroral oval as the magnetic field open-closed boundary in the particle precipitation observations (e.g., Boakes et al., 2008; Longden et al., 2010). Hubert et al. (2006) used the halfway point between b6 and 

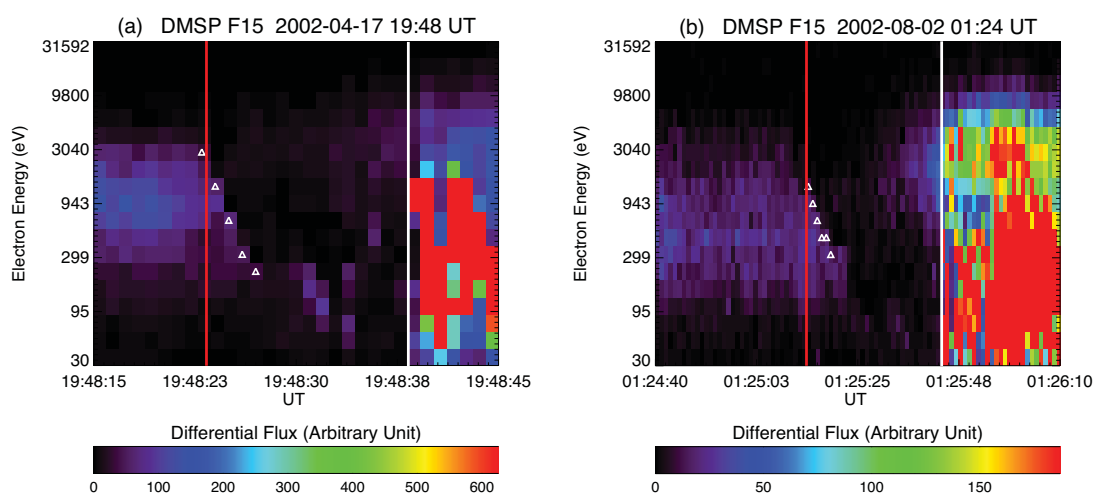

Figure 3. Polar rain electron dispersion observed by DMSP F15 at (a) 17 April 2002 19:48 UT and (b) 2 August 2002 01:24 UT. The white triangles, which indicate the highest energy detected at a given latitude, give a measure of the energy-latitude dispersion. The vertical red lines mark the location of the highest energy polar rain electrons in the dispersion region while the vertical white lines mark b6.

b5 (b5e and b5i are assumed to be identical) as the demarcation for open-closed boundary. Longden et al. (2010) found that the open-closed boundary determined from IMAGE ultraviolet (UV) Wideband Imaging Camera (WIC) and Spectrographic Imager SI-13 images are systematically poleward of DMSP b6 on the nightside by about $1-2^{\circ}$ in magnetic latitude (mlat), except perhaps near dawn for SI-13 open-closed boundary. Similarly, Boakes et al. (2008) found that IMAGE WIC open-closed boundary is systematically poleward of DMSP b6, except near dawn. The WIC (140-190 nm) and SI$13(135 \mathrm{~nm})$ emissions respond mostly to electron precipitation. Previous studies suggested correction algorithm for the magnetic field open-closed boundary obtained from auroral UV images based on the assumption that DMSP b6 gives the best estimate of this boundary (e.g., Boakes et al., 2008).

Irrespective of the auroral imagery and DMSP particle boundary comparisons above, there may be a reason to expect that the open-closed boundary is located poleward of b6. The magnetic field line open-closed boundary should correspond to the last open field line. In the presence of the dawn-dusk electric field, the electrons on the last open field line in the magnetotail would $\boldsymbol{E} \times \boldsymbol{B}$ to lower latitude as they travel to ionosphere. As a result, the dispersion region is typically equatorward of the open-closed boundary. The amount of the equatorward displacement would depend on the electron velocity (energy), the poleward component of $\boldsymbol{E} \times \boldsymbol{B}$, and the electron path length from the magnetotail $X$ line to the ionosphere. In the model run discussed in Sect. 2.2, partly because of the moderate $X$ line distance $\left(50 R_{\mathrm{E}}\right)$, the last open-field line is located just slightly less than $0.1^{\circ}$ poleward of the highest energy electrons in the energy-latitude dispersion region. Larger $X$ line distance would result in a larger displacement. Nevertheless, the highest energy electrons in the dispersion region can still serve as a better proxy for the open-closed boundary than b6, b5e, or b5i (the auroral oval poleward boundaries).
The highest energy electrons in the dispersion region are indicated with a vertical red line in Figs. 1, 2, and 3, which can be taken as the latitudinal lower bound of the openclosed boundary. As can be seen in Figs. 1 and 3, the vertical red line is poleward of b6, which is indicated by a vertical black and white line, respectively. For example, in Fig. 1b, b6 is located at $-71.5^{\circ}$ mlat whereas the red line is located at $-72.2^{\circ}$ mlat. Hence, the open-closed boundary is located at least $0.7^{\circ}$ or about $88 \mathrm{~km}$ more poleward than b6. In Fig. 1d, the open-closed boundary is located at least $1.2^{\circ}$ or $150 \mathrm{~km}$ poleward of $\mathrm{b} 6\left(\mathrm{~b} 6=74.3^{\circ}\right.$ mlat and the red line $=75.5^{\circ}$ mlat). The solar wind condition for the 16 June 1992 event shown in Fig. 1a and $\mathrm{b}$ is fairly moderate, IMF GSM $(X$, $Y, Z)=(7,-2,-2) \mathrm{nT},\left|V_{\mathrm{sw}}\right|=360 \mathrm{~km} \mathrm{~s}^{-1}, n_{\mathrm{sw}}=7 \mathrm{~cm}^{-3}$. There is no solar wind data for the 22 March 1984 event shown in Fig. 1c and d, but the magnetic activity indices indicate that the magnetic condition is moderately active, $\mathrm{Kp}=4+$, Dst $=-35 \mathrm{nT}$, and $\mathrm{AL}=-100 \mathrm{nT}$. For the two events in Fig. 3a and b, the open-closed boundary is located at least $0.7^{\circ}(88 \mathrm{~km})$ and $1.5^{\circ}(165 \mathrm{~km})$ poleward of b6, respectively. Our result is consistent with Sergeev and Bösinger (1993), which examines energetic (>30 keV) particle precipitation during moderately active times and concludes that the actual open-closed boundary may lie poleward of the auroral oval detected by auroral particles $(0.3-$ $20 \mathrm{keV})$.

However, the energy-latitude dispersion is not observed in every DMSP polar pass. It would be expected that the dispersion can only be observed when there is a significant $\boldsymbol{E} \times \boldsymbol{B}$ drift during the electron flight from the magnetotail $X$ line to the ionosphere. The conditions that would be favorable to observe the energy-latitude dispersion are strong dawn-dusk electric field, large $X$ line distance, and unusually low electron energy. The example shown in Fig. 1a and b, the 16 June 1992 event, suggests that the polar rain electron energy-latitude dispersion can be observed even when the solar wind driver is mild and IMF $B_{y}$ and $B_{z}$ are relatively 
small. The examples shown in Fig. 3 show that the dispersion can also be observed even when the polar rain electron energies are unusually high, on the order of a few $\mathrm{keV}$, but the IMF $B_{y}$ or $B_{z}$ is unusually large for these two events. When the dispersion is not observable, the open-closed boundary may be approximated by the location where polar rain ends near the nightside auroral oval or where the polar rain meets the auroral oval, in cases where they do meet.

\section{The electron overhang}

As can be seen in Figs. 1 and 3, equatorward of the polar rain dispersion regions, there is sometimes a gap, a region of little electron precipitation (Meng and Kroehl, 1977), followed by hot electrons (up to several $\mathrm{keV}$ or even a few tens of $\mathrm{keV}$ ) that are sometimes referred to as "electron overhang", which can be seen as a poleward extension of the nightside auroral oval electrons. The electrons in the overhang region have energies that are similar to those in the plasma sheet and higher than those in the polar rain. The overhang region often overlaps with the polar rain dispersion region such that the same flux tube can contain electrons from both regions as shown in Fig. 1. The overhang electrons are not well understood (Newell et al., 1996), although, in general, they are believed to originate from the plasma sheet (Shirai et al., 1997). The auroral UV open-closed boundary, which was sometimes found located poleward of b6 (e.g., Boakes et al., 2008; Longden et al., 2010), may correspond to the poleward boundary of the overhang electrons.

After reconnection, the supply of the solar wind electrons is shut off. So, after all the field-aligned polar rain electrons precipitate into the ionosphere, the loss cone would become empty, which may explain the gap region. Apparently, it takes some time for the plasma sheet electrons from the surrounding closed field lines to curvature and gradient drift into the newly closed field line and get pitch angle scattered. Consistent with the curvature and gradient drifts, which are energy dependent, the higher energy electrons would first drift into the flux tube. Then, as the flux tube continues to convect earthward (or equatorward in the ionosphere), the lower energy plasma sheet electrons would arrive. This would be consistent with the energy-latitude dispersion often seen in the overhang region seen in Figs. 1 and 3.

In order for the plasma sheet electrons to be observed by DMSP at low altitude, there has to be a mechanism for pitch-angle scattering. A leading mechanism for pitch-angle scattering is electron interactions with waves such as VLF whistler-mode chorus wave (e.g., Thorne, 2010; Summers et al., 1998; Gkioulidou et al., 2012; Wing et al., 2013). However, the wave-electron interaction may preferentially pitchangle scatter electrons with certain energies. We plan to calculate the energy dependence of the wave-electron interaction in a future study.

\section{Summary and conclusion}

APL-OPM successfully models the polar rain electron energy-latitude dispersion and demonstrates quantitatively that this dispersion results from the solar wind suprathermal electron entries in the last open-field line and $\boldsymbol{E} \times \boldsymbol{B}$ equatorward convection. Using APL-OPM, we find that the existing method for obtaining electron path length from the $X$ line to the ionosphere underestimates path lengths by at least $33 \%$. The best estimate is obtained by using the two highest energy electrons in the dispersion region.

The magnetic field line open-closed boundary is located slightly poleward of the highest energy electrons observed in the polar rain dispersion region, which, in turn is located poleward of b6, b5e, and b5i (DMSP auroral oval poleward boundaries). In our model run, partly because of the moderate $X$ line distance $\left(50 R_{\mathrm{E}}\right)$, the open-closed boundary is located just slightly less than $0.1^{\circ}$ poleward of the highest energy electrons in the energy-latitude dispersion region. Larger $X$ line distance would result in a larger displacement. Regardless, the location of the highest energy electrons would be a more accurate proxy for the open-closed boundary than $\mathrm{b} 6, \mathrm{~b} 5 \mathrm{i}$, or b5e. In the four DMSP cases examined, the open-closed boundary is located at least $0.7-1.5^{\circ}$ poleward of b6. Previous studies presented formulas to correct the open-closed boundary location obtained from the auroral UV imagery based on the assumption that $\mathrm{b} 6$ is the best available proxy. The present study suggests that these formulas need to be modified. However, the polar rain energy-latitude dispersion is not always observable, which may depend on the magnetotail electric field, the $X$ line distance, and the polar rain electron energy. For a future study, we will investigate statistically how often the energy-latitude dispersion can be observed. Finally, the energy-latitude dispersion seen in the electron overhang may result from the plasma sheet electron curvature and gradient drifts into the newly closed field line. The auroral UV open-closed boundary may correspond to the poleward boundary of this electron overhang.

Acknowledgements. The Air Force Research Laboratory has been helpful in the acquisition of DMSP SSJ4/SSJ5 and magnetometer data, as has the World Data Center in Boulder, Colorado. DMSP SSIES drift meter data were obtained at http://cindispace. utdallas.edu/DMSP/dmsp_data_at_utdallas.html. The SuperDARN convection pattern was obtained from Michael Ruohoniemi. All the derived data products in this paper are available upon request by email (simon.wing@jhuapl.edu). Simon Wing gratefully acknowledges support from NSF grants ATM-0802715 and AGS-1058456; NASA grants NNX13AE12G and NNX10AL01G. Yongliang Zhang acknowledges the support from NASA grant NNX10AL01G. Conor Francois, who was a summer intern at JHU/APL, contributed to the software.

Topical Editor L. Blomberg thanks one anonymous referee for his/her help in evaluating this paper. 


\section{References}

Alexeev, I. V., Sergeev, V., Owen, C. J., Fazakerley, A., Lucek, E., and Réme, H.: Remote sensing of a magnetotail reconnection $X$ line using polar rain electrons, Geophys. Res. Lett., 33, L19105, doi:10.1029/2006GL027243, 2006.

Baker, K. B. and Wing, S.: A new magnetic coordinate system for conjugate studies at high latitudes, J. Geophys. Res., 94, 91399143, doi:10.1029/JA094iA07p09139, 1989.

Boakes, P. D., Milan, S. E., Abel, G. A., Freeman, M. P., Chisham, G., Hubert, B., and Sotirelis, T.: On the use of IMAGE FUV for estimating the latitude of the open/closed magnetic field line boundary in the ionosphere, Ann. Geophys., 26, 2759-2769, doi:10.5194/angeo-26-2759-2008, 2008.

Cowley, S. W. H. and Owen, C. J.: A simple illustrative model of open flux tube motion over the dayside magnetopause, Planet. Space Sci., 37, 1461-1475, 1989.

Dungey, J. W.: Interplanetary magnetic fields and the auroral zones, Phys. Rev. Lett., 6, 47-48, 1961.

Fairfield, D. H. and Scudder, J. D.: Polar rain: Solar coronal electrons in the Earth's magnetosphere, J. Geophys. Res., 90, 40554068, doi:10.1029/JA090iA05p04055, 1985.

Gkioulidou, M., Wang, C.-P., Wing, S., Lyons, L. R., Wolf, R. A., and Hsu, T.-S.: Effect of an MLT dependent electron loss rate on the magnetosphere-ionosphere coupling, J. Geophys. Res., 117, A11218, doi:10.1029/2012JA018032, 2012.

Hardy, D. A., Gussenhoven, M. S., Riehl, K., Burkhardt, R., Heinemann, N., and Schumaker, T.: The characteristics of polar-cap precipitation and their dependence on the interplanetary magnetic field and the solar wind, in: Solar Wind-Magnetosphere Coupling, edited by: Kamide, Y. and Slavin, J. A., 575-604, Terra, Tokyo, 1986.

Hill, T. W. and Reiff, P. H.: Evidence of magnetospheric cusp proton acceleration by magnetic merging at the dayside magnetopause, J. Geophys. Res., 82, 3623-3628, 1977.

Hubert, B., Milan, S. E., Grocott, A., Blockx, C., Cowley, S. W. H., and Gérard, J.-C.: Dayside and nightside reconnection rates inferred from IMAGE FUV and Super Dual Auroral Radar Network data, J. Geophys. Res., 111, A03217, doi:10.1029/2005JA011140, 2006.

Longden, N., Chisham, G., Freeman, M. P., Abel, G. A., and Sotirelis, T.: Estimating the location of the open-closed magnetic field line boundary from auroral images, Ann. Geophys., 28, 1659-1678, doi:10.5194/angeo-28-1659-2010, 2010.

Meng, C.-I. and Kroehl, H. W.: Intense uniform precipitation of low-energy electrons over the polar cap, J. Geophys. Res., 82, 2305-2313, doi:10.1029/JA082i016p02305, 1977.

Newell, P. T., Wing, S., Meng, C.-I., and Sigillito, V.: The auroral oval position, structure, and intensity of precipitation from 1984 onward: An automated on-line data base, J. Geophys. Res., 96, 5877-5882, doi:10.1029/90JA02450, 1991.
Newell, P. T., Feldstein, Y. I., Galperin, Y. I., and Meng, C.-I.: Morphology of nightside precipitation, J. Geophys. Res., 101, 10737-10748, doi:10.1029/95JA03516, 1996.

Pedersen, A., Cattell, C. A., Fälthammar, C.-G., Knott, K., Lindqvist, P.-A., Manka, R. H., and Mozer, F. S.: Electric fields in the plasma sheet and plasma sheet boundary layer, J. Geophys. Res., 90, 1231-1242, doi:10.1029/JA090iA02p01231, 1985.

Ruohoniemi, J. M. and Greenwald, R. A.: Statistical patterns of high-latitude convection obtained from Goose Bay HF radar observations, J. Geophys. Res., 101, 21743-21763, doi:10.1029/96JA01584, 1996.

Sergeev, V. A. and Bösinger, T.: Particle dispersion at the nightside boundary of the polar cap, J. Geophys. Res., 98, 233-241, doi:10.1029/92JA01667, 1993.

Shirai, H., Maezawa, K., Fujimoto, M., Mukai, T., Yamamoto, T., Saito, Y., Kokubun, S., and Kaya, N.: Drop-off of the polar rain flux near the plasma sheet boundary, J. Geophys. Res., 102, 2271-2278, 1997.

Spreiter, J. R. and Stahara, S. S.: Magnetohydrodynamic and gasdynamic theories for planetary bow waves, in: Collisionless Shocks in the Heliosphere: Reviews of Current Research, Geophys. Monogr. Ser., Vol. 35B, edited by: Tsurutani, T. and Stone, R. G., 85-107, AGU, Washington, D.C., 1985.

Summers, D., Thorne, R. M., and Xiao, F.: Relativistic theory of wave-particle resonant diffusion with application to electron acceleration in the magnetosphere, J. Geophys. Res., 103, 20487 20500, doi:10.1029/98JA01740, 1998.

Thorne, R. M.: Radiation belt dynamics: The importance of wave-particle interactions, Geophys. Res. Lett., 37, L22107, doi:10.1029/2010GL044990, 2010.

Tsyganenko, N. A. and Stern, D. P.: Modeling the global magnetic field of the large-scale Birkeland current systems, J. Geophys. Res., 101, 27187-27198, doi:10.1029/96JA02735, 1996.

Wing, S., Newell, P. T., and Onsager, T. G.: Modeling the entry of magnetosheath electrons into the dayside ionosphere, J. Geophys. Res., 101, 13155-13167, doi:10.1029/96JA00395, 1996.

Wing, S., Newell, P. T., and Rouhoneimi, J. M.: Double cusp: Model prediction and observational verification, J. Geophys. Res., 106, 25571-25593, doi:10.1029/2000JA000402, 2001.

Wing, S., Gkioulidou, M., Johnson, J. R., Newell, P. T., and Wang, C.-P.: Auroral particle precipitation characterized by the substorm cycle, J. Geophys. Res.-Space, 118, 1022-1039, doi:10.1002/jgra.50160, 2013.

Zhang, Y., Paxton, L. J., and Kil, H.: Nightside polar rain aurora boundary gap and its applications for magnetotail reconnection, J. Geophys. Res., 116, A11214, doi:10.1029/2011JA016884, 2011. 